\title{
Influencia del sistema HACCP en la mejora continua de la línea de comidas preparadas en un autoservicio de Lima Metropolitana (Perú)
}

RECIBIDO:29/11/2017 ACEPTADO: 11/05/2018

Gilda Rosas Aparicio ${ }^{1}$

\begin{abstract}
RESUMEN
La presente investigación pretende determinar de qué manera la gestión de la calidad, basado en el sistema HACCP (Análisis de Riesgos y de Puntos Críticos de Control), influye en la mejora continua de la Línea de Comidas Preparadas en un autoservicio de Lima Metropolitana al año 2015. Para ello, se abordó la mejora continua desde dos perspectivas: Responsabilidad del personal manipulador de alimentos y las condiciones de proceso y fabricación e instalaciones sanitarias. El tipo de investigación fue preexperimental; la muestra de estudio estuvo conformada por 16 personas que laboran en la Línea de Comidas Preparadas de un autoservicio de Lima Metropolitana. Se concluye que el sistema HACCP sí ha influido de manera significativa en la mejora continua, así como también, en la responsabilidad de las personas que manipulan alimentos y en las condiciones de proceso y fabricación e instalaciones sanitarias del autoservicio.
\end{abstract}

Palabras-claves: Sistema HACCP; mejora continua; manipulación de alimentos.

INFLUENCE OF THE HACCP SYSTEM ON THE CONTINUOUS IMPROVEMENT OF THE LINE OF READY MEALS IN A SELFSERVICE STORE OF Lima Metropolitan AREa (PERU)

\section{ABSTRACT}

This research aims to determine how the quality management, based on the HACCP system (Hazard Analysis and Critical Control Points), influences on the continuous improvement of the Line of Ready Meals in a self-service store of Lima Metropolitan Area to 2015. For this reason, the continuous improvement was studied from two perspectives: Responsibility of food handling personnel and the conditions of process and manufacturing and sanitary facilities. The type of research was pre-experimental; the sample of study was conformed by 16 people who work in the Line of Ready Meals of a self-service store of Lima Metropolitan Area. It is concluded that the HACCP system has had a significant influence on continuous improvement, as well as, on the responsibility of food handlers and the conditions of processing and manufacturing and sanitary facilities of self-service store.

Keywords: HACCP system; continuous improvement; food handling.

\section{INTRODUCCIÓN}

Griffith, Jackson y Lues (2017) consideran que la seguridad alimentaria es una de las mayores preocupaciones de la humanidad en la actualidad. Los autores señalan que cada año, aproximadamente más de 600 millones de individuos en el mundo (en la proporción de 1 de cada 10) padecen enfermedades transmitidas por los alimentos; de los cuales, 420000 son niños que fallecen por algún riesgo en particular, ya que "Ios alimentos elaborados bajo condiciones no adecuadas pueden contener bacterias, virus, parásitos o sustancias químicas nocivas que causan más de 200 enfermedades transmitidas por alimentos"(Castañeda, Fuentes y Peñarrieta, 2016, p. 197). Muñoz (2003) señalaque en América Latina se han reportado varios casos de contaminación de los alimentos en centros educativos públicos y hospitales, los cuales han sido tratados por investigaciones realizadas en Brasil, Cuba, Chile y Panamá. En el Perú, al año 2008, se registraban anualmente cerca de 1,700,000 casos de enfermedades infecciosas intestinales, constituyendo la tercera causa de morbilidad en el país; dichas enfermedades guardaban relación con una inadecuada manipulación de los alimentos en el hogar, micro y pequeñas empresas dedicadas al expendio de alimentos, entre otros (Ministerio de Salud, 2016).

La Organización de las Naciones Unidas para la Agricultura y la Alimentación (2008) señala que dentro de la cadena alimentaria existen tres agentes responsables de garantizar que los alimentos estén libres de enfermedades. Dichos responsables son:

- El gobierno: Es responsable de cuidar la salud pública y obligar a todos los demás miembros de la cadena alimentaria a procurar la calidad de los alimentos.

- Los consumidores: Tienen la obligación de "observar las buenas prácticas de higiene durante la manipulación y almacenar los alimentos de forma adecuada según las recomendaciones del fabricante que figuren en la etiqueta" (Organización de las Naciones Unidas para la Agricultura y la Alimentación, 2008, p. 6).

1 E-mail: grosas@candymarket.com.pe

ORCID: 0000-0002-0745-115X

Ingeniera Pesquera de la Universidad Nacional del Callao (Lima-Perú). Consultora en calidad sanitaria y Auditora Líder Freelance en BPM y HACCP. 
- La industria alimentaria: Constituida por los productores, fabricantes, comercializadores al por menor y responsables de manipular los alimentos. Tienen la responsabilidad de garantizar que los alimentos que ofrecen no contengan enfermedades de transmisión y cumplir con la normativa vigente.

Según Dal Prà (2013)se considera como antecedentes del sistema de Análisis de Riesgos y de Puntos Críticos de Control (conocido por sus siglas en inglés como HACCP) a las investigaciones de Van Oyen, Meyer; y posteriormente Wilson y Mossel, quienes consideraron que la seguridad de los productos alimenticios no debía depender del control analítico, y por dicha razón, observaron la cadena productiva de los alimentos y concluyeron que se debía aplicar medidas e intervenciones en el proceso productivo, almacenamiento y comercialización de los mismos. Además, la misma autora señala que el sistema HACCP se ideó a fines de los años '60 con la finalidad de garantizar la seguridad de los alimentos que eran suministrados a los astronautas de la Administración Nacional de la Aeronáutica y del Espacio (conocida por sus siglas en inglés como NASA); expandiéndose a la industria de los Estados Unidos de América a inicios de la década de 1970 y a Europa desde los años '90. Lo anterior es complementado por Zapata (2011), quien señala que la primera industria estadounidense donde se aplicó el sistema HACCP fue la de conservas enlatadas de baja acidez (desde 1973), extendiéndose luego a los hidrobiológicos y las carnes.

Filippi (2014) sostiene que el sistema HACCP es una herramienta empresarial que tiene un fundamento científico, cuya finalidad es detectar peligros específicos y emprender medidas para controlarlos, todo ello en beneficio de la seguridad alimentaria. Es decir, el sistema HACCP se enfoca en prevenir posibles peligros dentro de la cadena de producción de alimentos, en lugar de solucionar problemas con productos alimenticios terminados. Además, el autor señala que la implementación de un sistema HACCP requiere de un estudio previo conformado por las siguientes cuatro etapas:

- Descripción en profundidad del producto (o conjunto de productos), respecto a: composición estructural, insumos químicos empleados, condiciones mínimas de almacenamiento.

- Usos que el consumidor o público objetivo le da al producto alimenticio que forma parte del proceso.

- Elaboración de un flujograma, en el cual se detallen todas las etapas desde la recepción de las materias primas hasta la distribución a los diferentes canales de comercialización.

- Cada etapa del flujograma elaborado debe ser contrastado con la realidad, ello para emprender las medidas correctivas necesarias.

Además, según Butrón (2012) el sistema HAC$\mathrm{CP}$ puede ser implementado en cualquier tipo de organización empresarial, sin importar el tipo de alimento que produzcan, siempre y cuando ello responda a sus necesidades. No obstante, la implementación de un sistema HACCP puede verse amenazada por tres factores que reducirian su eficiencia. En un estudio realizado por Panisello, Quantick y Knowles (2001), citado en Casolani y Del Signore, (2016) los autores explican que dichos factores son los siguientes:

- Principio de peligro perdido: No hay control de riesgos ya que éstos no son percibidos.

- Principio de riesgo perdido: No se considera la existencia de riesgos, a pesar de que los peligros están plenamente identificados.

- Principio de prevención perdida: Existe una plena identificación de peligros y riesgos, pero éstos no son adecuadamente analizados y además, existe carencia de procedimientos de control.

Filippi (2014) señala que el sistema HACCP se encuentra fundamentado en siete principios (Véase figura 1), cuyo conocimiento por parte de los colaboradores de una empresa alimenticia resulta vital para incrementar los niveles de seguridad alimentaria.

Por su parte, Panisello y Quantick (2001) sostienen que la implementación exitosa de un sistema HACCP, además de los siete principios, debe estar fundamentado bajo cuatro pilares, los cuales son:

- Compromiso de la Alta Dirección: La implementación exitosa de un sistema HACCP requiere del trabajo mutuo entre la Alta Dirección y los colaboradores de la organización empresarial.

- Educación y entrenamiento: La implementación del sistema HACCP previamente requiere que el personal involucrado directamente esté debidamente capacitado. Con dicha finalidad, la capacitación puede constar de vídeos, charlas presenciales de entrenamiento, elaboración de manuales, entre otros).

- Disponibilidad de recursos: La planificación del sistema HACCP debe ser constantemente monitoreada, por dicha razón, requiere que 


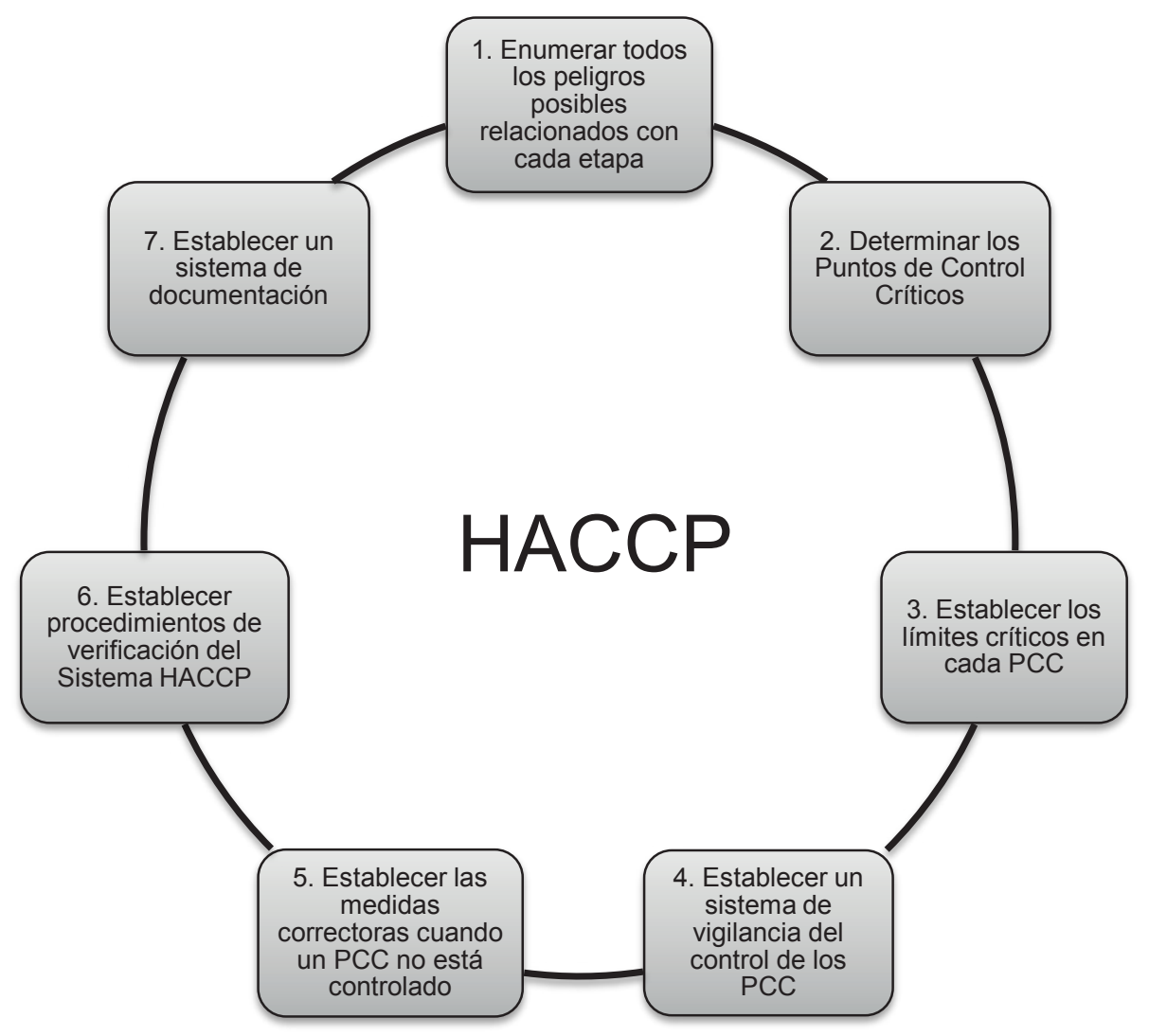

Figura 1. Principios del sistema HACCP

Fuente. Filippi (2014)

Elaboración propia

la organización empresarial invierta mediante desembolsos de recursos financieros, gestión del tiempo, mano de obra y apoyo en el entrenamiento del personal.

- Presión externa: Las organizaciones que conforman la industria alimenticia muchas veces no implementan un sistema HACCP por decisión propia, sino por obligación de grupos de presión, tales como: Gobierno Central, clientes, organismos reguladores, entre otros.

El sistema HACCP es considerado el protocolo de referencia cuando se trata de asegurar la inocuidad de los alimentos, razón por la cual ha sido adoptado como un programa de obligatorio cumplimiento en casi todos los países del mundo (Gutiérrez, Pastrana y Castro, 2011). Desde el 2008, el HACCP ha incrementado su importancia debido a los principales problemas ocurridos en la manipulación de alimentos, tales como alimentos envenenados, objetos extraños y deficiente calidad sanitaria, que son los mayores y más serios problemas resultantes del proceso de producción, generándose así una nueva cultura de cuidado de los alimentos(Souza, Novo, Fernandes y Kawamoto, 2016). Pero dicha cultura no se desarrolla por sí misma, ya que los recursos humanos son uno de los componentes clave del sistema HACCP y quienes serán el factor determinante del éxito de su implementación (García, Quiñones y Parra, 2014).

Dado que el HACCP es un sistema reconocido y eficaz, proporciona a los clientes la confianza en la seguridad del proceso e indica que la empresa que lo aplica es profesional y toma en serio sus responsabilidades (Mortimore y Wallace, 2001). La integración de los principios del HACCP, en la venta al por menor y el servicio de comidas, en el quehacer diario y el compromiso de la gerencia en materia de seguridad alimentaria son los pilares de un sistema HACCP eficaz en este sector productivo(ASQ Food, Drug, and Cosmetic Division, 2003). De acuerdo a la normativa del Reglamento Sanitario de Funcionamiento de Autoservicios de Alimentos y Bebidas (Ministerio de Salud, 2002) se ha podido identificar no conformidades en infraestructura y operaciones, halladas en inspecciones higiénico sanitarias a diversos autoservicios de Lima Metropolitana por parte de la Autoridad de Salud Municipal de cada distrito. Generalmente las clausuras y sanciones de 
los locales son informadas a la población por medios de comunicación masiva(El Comercio, 2015).

A inicios del año 2015 el autoservicio en evaluación presentó un cumplimento de $74 \%$ en los prerrequisitos (Buenas Prácticas de Manufactura y Programa de Higiene y Saneamiento) y $72 \%$ en el sistema HAC$\mathrm{CP}$ como resultados de la inspección sanitaria, obteniendo la calificación de nivel de cumplimiento bajo con una frecuencia de medición mensual. Por otra parte, presentó un cumplimiento del $86 \%$ lo cual lo califica como regular, en la primera auditoria higiénico sanitaria con una frecuencia de medición semestral y un $45 \%$ de cumplimiento con respecto a los muestreos microbiológicos en una etapa inicial.

El objetivo principal de la presente investigación es determinar de qué manera el sistema HACCP influye en la mejora continua de la Línea de Comidas Preparadas en un autoservicio de Lima Metropolitana al año 2015. Además, dicho objetivo principal ha sido desagregado en dos objetivos específicos, buscando evaluar de qué manera el Sistema HACCP influye en la responsabilidad del personal manipulador de alimentos, y en las condiciones de proceso y fabricación e instalaciones sanitarias de la Línea de Comidas Preparadas en la organización empresarial de estudio.

\section{METODOLOGÍA}

La presente investigación fue de tipo preexperimental y se aplicó el diseño de prueba/posprueba con un solo grupo por las siguientes razones:

- Según Hernández, Fernández y Baptista (2014), "a un grupo se le aplica una prueba previa al estímulo o tratamiento experimental, después se le administra el tratamiento y finalmente se le aplica una prueba posterior al estímulo" (p. 141). Según los mismos autores, el diseño de investigación se diagrama de la siguiente manera:

$$
G \quad 0_{1} \quad X \quad 0_{2}
$$

Donde:

G: Grupo de sujetos (16 participantes)

$X$ : Tratamiento, estímulo o condición experimental (implementación del sistema HACCP)

$0_{1}$ : Medición realizada antes de la implementación del sistema HACCP

$\mathrm{O}_{2}$ : Medición realizada luego de la implementación del sistema HACCP
- Complementando lo expresado en el diagrama precedente, se manipuló la variable independiente (sistema HACCP), puesto que se realizó una primera medición en febrero de 2015 (preprueba), luego los participantes fueron capacitados en todos aquellos temas relacionados con la implementación del sistema HACCP y finalmente, se realizó una segunda medición en noviembre del mismo año (posprueba) con la finalidad de analizar los cambios que se dieron durante dicho intervalo de tiempo. La muestra de estudio, debido a su pequeño tamaño, abarcó a toda la población, la misma que estuvo conformada por dieciséis personas que laboran en la Sección de Comidas Preparadas de un autoservicio limeño. Por motivos de seguridad, se ha mantenido en reserva el nombre de la empresa.

A pesar de tratarse de una muestra estadística pequeña (menor a 30 casos), según Rodríguez, Pierdant y Rodríguez (2014), ello no impide la realización ni resta importancia a la prueba de hipótesis.La prueba estadística seleccionada para este estudio fue la T de Student, ya que según Hernández, Fernández y Baptista (2014), "se utiliza para comparar los resultados de una preprueba con los resultados de una posprueba en un contexto experimental" ( $\mathrm{p}$. 311).

Los instrumentos de recolección de datos que empleó la presente investigación fueron las listas de chequeo utilizadas en las inspecciones sanitarias mensuales y auditorías higiénico sanitarias semestrales. Los datos obtenidos fueron procesados y analizados con el software estadístico IBM SPSS Versión 23 con la finalidad de que la información sea entendida por profesionales de diversas disciplinas académicas.

\section{RESULTADOS}

El objetivo de las inspecciones sanitarias en un autoservicio es uniformizar los criterios de evaluación y calificación de los hallazgos en las secciones de perecibles por parte de los inspectores del área de Aseguramiento de la Calidad de la organización.La frecuencia de realización de las inspecciones sanitarias es mensual y se basan en los siguientes documentos que rigen el desempeño en materia de calidad:

- Manual de Saneamiento

- Manual HACCP

- Manual de Higiene 
- Normas de Vida Útil Límite para recepción y retiro de venta

- Procedimiento de Rotación de Productos a Granel

- Norma de Control de Tiempo de Retiro

- Lineamiento de Evaluación de las Condiciones de Entrega de Mercadería

Durante la inspección sanitaria se evaluó la infraestructura, equipos, mobiliario, utensilios y conocimientos de calidad sanitaria por parte de los manipuladores. A continuación, en la figura 2 se exponen los resultados de cuatro meses del año 2015 para ver la tendencia del desempeño de la línea de comidas preparadas en lo concerniente al Programa de Buenas Prácticas de Manipulación, el Programa de Higiene y Saneamiento y el Sistema HACCP.

De acuerdo a la anterior figura 2, se evidencióen la primera inspección sanitaria una serie de no conformidades las cuales se vieron reflejadas en el porcentaje obtenido de cumplimiento, $74 \%$ en BPM-POES (Buenas Prácticas de Manipulación-Procedimientos Operativos Estandarizados) y $72 \%$ en HACCP.

Para revertir los resultados de la primera inspección sanitaria el equipo de trabajo encargado del siste- ma HACCP necesitó conocer perfectamente cuál era el punto de partida. Esto se consigue fácilmente si todos los miembros del equipo han participado en la inspección inicial, incluyendo la revisión de los procedimientos documentados en funcionamiento, los aspectos medioambientales, la situación de las BPM, la disponibilidad de recursos y del estado de la formación(Mortimore y Wallace, 2001).

La organización empresarial de estudio, interesada en la revisión del desempeño del Sistema de Gestión de Calidad y comprometidos con la mejora continua de sus procesos tiene establecido, a través del Área de Aseguramiento de la Calidad, un Programa Anual de Auditorias del Sistema de gestión BPM/HACCP en su cadena de Autoservicios. Luego de cada auditoría se presentó un informe que daba a conocer los resultados de las auditorías programadas por el Área de Aseguramiento de la Calidad de la Organización, con frecuencia semestral, donde se puso énfasis en los puntos críticos de control (recepción de productos, almacenamiento, desinfección, pre cocción, cocción, exhibición)a través de la revisión de los informes de las inspecciones sanitarias mensuales realizadas por los inspectores de calidad a fin de evidenciar las mejoras y logros alcanzados en el autoservicio. Este tipo de auditorías garantizaron que los puntos críticos de control seleccionados estén siempre bajo control.

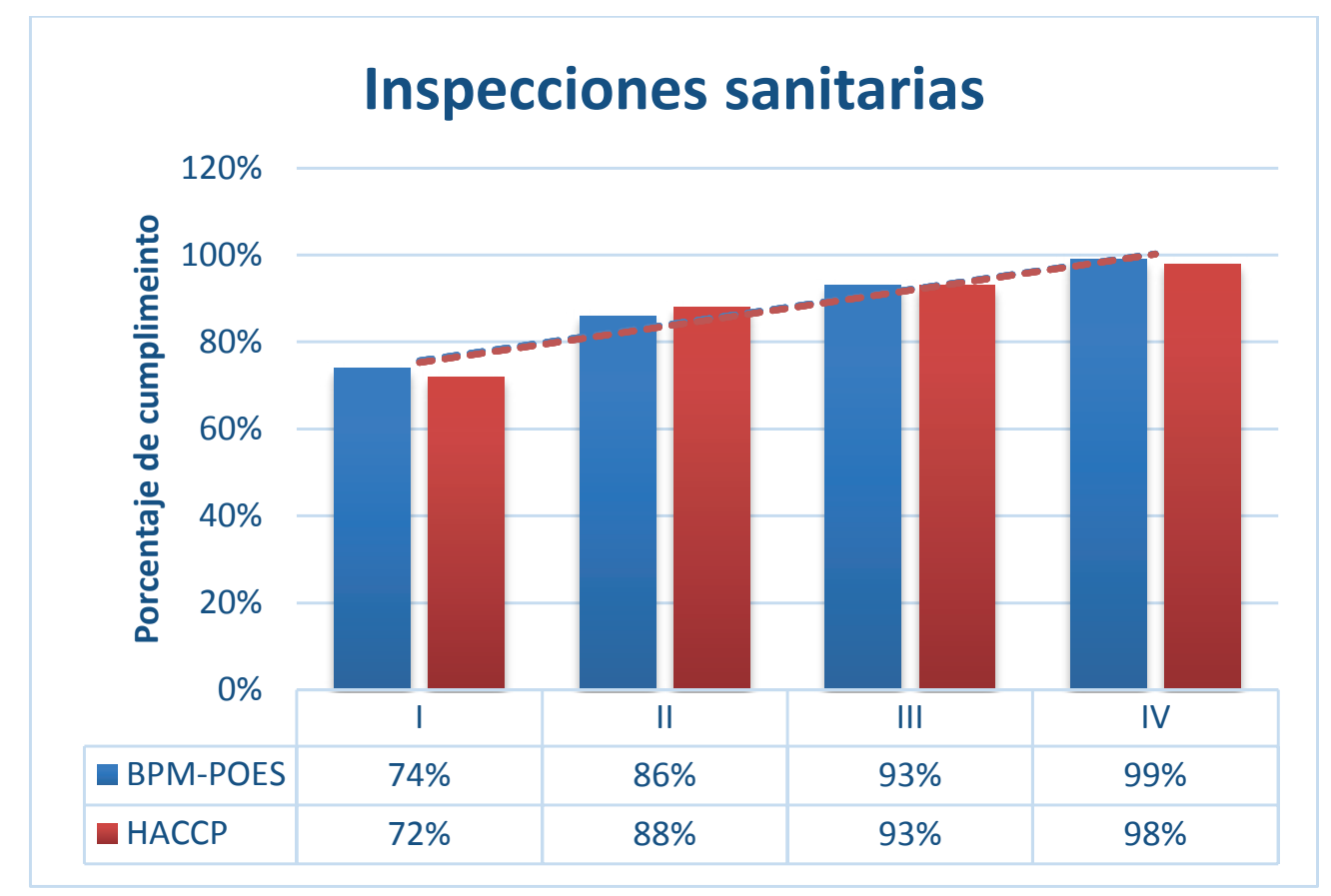

Figura 2. Inspecciones sanitarias internas

Fuente y elaboración propia 
Las auditorias estuvieron a cargo de empresas especializadas reconocidas dentro del medio, los documentos utilizados como referencia son:

- Código Internacional Recomendado de Prácticas-Principios Generales de Higiene de los Alimentos CAC/RCP 1-1969, Rev. 4 (2003).

- Sistema de Análisis de Peligros y de Puntos Críticos de Control (HACCP)-Directrices para su Aplicación. Anexo al CAC/RCP1.

- Reglamento Sanitario de Funcionamiento de Autoservicios de Alimentos y Bebidas R.M. N¹653-2002-SA/DM.

- Manual HACCP y Manual de Saneamiento Vigentes.

- Adicionalmente se auditan los documentos aprobados por Aseguramiento de Calidad de la Corporación: Procedimiento de Condiciones de Entrega de Mercadería, Norma de Control de Tiempos de Retiro, Norma de Recojo M7 y Norma de Rotación de Productos a Granel. Asimismo, se audita registros correspondientes.

En las auditorías se evaluaron también los resultados deanálisis microbiológicos efectuados a superficies vivas, inertes, ambientes y productos elaborados. El objetivo fue verificar que el proceso se encontraba bajo control y que se elaboraba un producto aceptable.En el caso de los peligros microbiológicos o químicos, el mayor problema relacionado con la inspección del $100 \%$ era que no quedaría nada para la venta, ya que los análisis químicos y microbiológicos son casi siempre destructivos y esto obligó a la utilización de planes de muestreo.Por tal motivo, la organización tomó muestras aleatorias en la línea de producción con frecuencia anual, la segunda toma correspondía al seguimiento de los primeros. La organización subcontrató los servicios de muestreo y análisis de las principales empresas reconocidas dentro del medio.

El contraste de hipótesis se realizó empleando el software estadístico IBM SPSS Versión 23, por el cual, se prueba que el Sistema HACCP sí influye en la mejora continua de la Línea de Comidas Preparadas en un autoservicio de Lima Metropolitana durante el año 2015, ya que el valor de $t=-2.792$ es menor que la significancia (véase tabla 1).

Con respecto al primer objetivo específico, se determina que el Sistema HACCP sí influye en la responsabilidad del personal manipulador de alimentos en un autoservicio de Lima Metropolitana durante el año 2015, ya que el valor de $t=-2.345$ es menor que la significancia, tal como se puede apreciar en la siguiente tabla 2 :

Por otra parte,para el segundo objetivo específico, en la tabla 3 se verifica que el Sistema HACCP sí influye en las condiciones de proceso y fabricación e instalaciones sanitarias en un autoservicio de Lima Metropolitana durante el año 2015, ya que el valor de $t=-2.570$ es menor que la significancia.

Tabla 1. Influencia del Sistema HACCP en la mejora continua

\begin{tabular}{|c|c|c|c|c|c|c|c|c|c|}
\hline & & \multicolumn{5}{|c|}{ Diferencias relacionadas } & \multirow{3}{*}{$\mathbf{t}$} & \multirow{3}{*}{ gl } & \multirow{3}{*}{ Sig. (bilateral) } \\
\hline & & \multirow{2}{*}{ Media } & \multirow{2}{*}{ Desviación típ. } & \multirow{2}{*}{$\begin{array}{c}\text { Error típ. de } \\
\text { la media }\end{array}$} & \multicolumn{2}{|c|}{$\begin{array}{c}95 \% \text { Intervalo de confian- } \\
\text { za para la diferencia } \\
\end{array}$} & & & \\
\hline & & & & & Inferior & Superior & & & \\
\hline Par 1 & $\begin{array}{l}\text { Mejora continua - (Antes) } \\
\text { Mejora continua - (Después) }\end{array}$ & $\mid-, 36047$ & 1,19718 & 12910 & $-0,61714$ & $-0,10379$ & $-2,792$ & 85 & 0,006 \\
\hline
\end{tabular}

Fuente. Elaboración propia.

Tabla 2. Influencia del Sistema HACCP en la responsabilidad del personal manipulador de alimentos

\begin{tabular}{|c|c|c|c|c|c|c|c|c|c|}
\hline & & \multirow{2}{*}{\multicolumn{5}{|c|}{ Diferencias relacionadas }} & \multirow{4}{*}{$\mathbf{t}$} & \multirow{4}{*}{ gl } & \multirow{4}{*}{ Sig. (bilateral) } \\
\hline & & & & & & & & \\
\hline & & \multirow{2}{*}{ Media } & \multirow{2}{*}{$\begin{array}{l}\text { Desviación } \\
\text { típ. }\end{array}$} & \multirow{2}{*}{$\begin{array}{c}\text { Error típ. } \\
\text { de la media }\end{array}$} & \multicolumn{2}{|c|}{$\begin{array}{c}95 \% \text { Intervalo de confianza } \\
\text { para la diferencia }\end{array}$} & & & \\
\hline & & & & & Inferior & Superior & & & \\
\hline \multirow[b]{2}{*}{ Par 1} & $\begin{array}{l}\text { Responsabilidad del personal ma- } \\
\text { nipulador de alimentos - (Antes) }\end{array}$ & \multirow[b]{2}{*}{,- 66667} & \multirow[b]{2}{*}{,98473 } & \multirow[b]{2}{*}{,28427 } & \multirow[b]{2}{*}{$-1,29234$} & \multirow[b]{2}{*}{$-0,04100$} & \multirow[b]{2}{*}{$-2,345$} & \multirow[b]{2}{*}{11} & \multirow[b]{2}{*}{0,0039} \\
\hline & $\begin{array}{l}\text { Responsabilidad del personal ma- } \\
\text { nipulador de alimentos-(Después) }\end{array}$ & & & & & & & & \\
\hline
\end{tabular}

Fuente. Elaboración propia. 
Tabla 3. Influencia del Sistema HACCP en lascondiciones de proceso y fabricación e instalaciones sanitarias

\begin{tabular}{|c|c|c|c|c|c|c|c|c|c|}
\hline & \multicolumn{5}{|c|}{ Diferencias relacionadas } & \multirow{3}{*}{$\mathbf{t}$} & \multirow{3}{*}{ gl } & \multirow{3}{*}{ Sig. (bilateral) } \\
\hline & & \multirow[t]{2}{*}{ Media } & \multirow[t]{2}{*}{ Desviación típ. } & \multirow{2}{*}{$\begin{array}{c}\text { Error típ. de } \\
\text { la media }\end{array}$} & \multicolumn{2}{|c|}{\begin{tabular}{|c|}
$95 \%$ Intervalo de confianza \\
para la diferencia
\end{tabular}} & & & \\
\hline & & & & & Inferior & Superior & & & \\
\hline Par 1 & $\begin{array}{l}\text { Condiciones de proceso e insta- } \\
\text { laciones sanitarias - (Antes) } \\
\text { Condiciones de proceso e insta- } \\
\text { laciones sanitarias- (Después) }\end{array}$ &,- 49020 & 1,36195 & , 19071 & $-0,87325$ & $-0,10714$ & $-2,570$ & 50 & 0,0013 \\
\hline
\end{tabular}

Fuente: Elaboración Propia

\section{DISCUSIÓN}

La garantía sanitaria de los alimentos tiene que ser una exigencia innegociable, por parte de los consumidores, e irrenunciable, por parte de los responsables de las empresas de preparación y venta de comidas (Ruiz, 2003).Los requisitos previos del sistema HACCP precisan de una gestión y evaluación continuada. Se deben utilizar auditorías e inspecciones para evaluar la eficacia de los mismos y para garantizar la mejora continua y el control de los peligros alimentarios (ASQ Food, Drug, and Cosmetic Division, 2003).

Los resultados obtenidos en la presente investigación son respaldados por el estudio realizado por Casolani y Del Signore (2016), quienes señalan que los beneficios de la implementación de un sistema HACCP son mayores que los costos y además como complemento, añaden que no se puede lograr la seguridad alimentaria sin la implementación del HACCP.

En la presente investigación se pudo comprobar que existía una fuerte influencia del sistema HACCP en la mejora continua, la cual también es compartidacon el estudio realizado por Trafiałek (2017), quien señala que existe una fuerte asociación entre la calidad (mejora continua) y la seguridad alimentaria (sistema HACCP), a pesar de que, según la autora, los siete principios del HACCP pueden causar problemas para mantener el sistema, su cumplimiento es necesario para garantizar la seguridad alimentaria. Además, Lavilla y Arizábal (2011) añaden que la relación entre HACCP y mejora continua (gestión de calidad) es complementaria y de carácter gerencial, ya que el compromiso con la seguridad alimentaria mejora la imagen de la organización empresarial ante los consumidores, constituyéndose en una ventaja competitiva. Por otra parte, los anteriores planteamientos se contradicen con la investigación de Castañeda, Fuentes y Peñarrieta (2016) quienes indican que si bien el HACCP goza de aceptación a nivel mundial para la prevención de peligros y garantizar la seguridad alimentaria, ello no guarda ningún tipo de relación con la gestión de la calidad en la producción de alimentos.

Se pudo corroborar que el sistema HACCP influye en la responsabilidad del personal manipulador de alimentos, dicha afirmación es respaldada y ampliada por la investigación de Griffith, Jackson y Lues (2017), quienes añaden que la cultura de la seguridad alimentaria no solo es responsabilidad del personal que tiene contacto directo con los alimentos, sino también de todos los miembros que conforman una organización empresarial y considera que dicho proceso debe ser liderado por la Alta Dirección. García, Quiñones y Parra (2014) añaden que el personal manipulador de alimentos debe tener las siguientes competencias: Buena presentación y estado de salud óptimo, ser un profesional ético y tener un dominio general de los procedimientos de higiene, limpieza y desinfección.

\section{CONCLUSIONES}

Las conclusiones de la presente investigación son las siguientes:

- El sistema HACCP sí ha influido en la mejora continua de la Línea de Comidas Preparadas en un autoservicio de Lima Metropolitana durante el año 2015.

- Del mismo modo, también ha habido una influencia del sistema HACCP en la responsabilidad del personal manipulador de alimentos, así como también en las condiciones de proceso e instalaciones sanitarias de la organización empresarial donde se realizó la investigación.

- Cada etapa de la implementación de un sistema HACCP debe estar debidamente documentada y ser de conocimiento, no solo del personal involucrado, sino también de toda la organización. 


\section{REFERENCIAS BIBLIOGRÁFICAS}

ASQ Food, Drug and Cosmetic Division. (2003). HACCP Manual del auditor de calidad. Zaragoza: Acribia, S.A.

Butrón, D. (2012). Propuesta de implementación de un sistema HACCPpara empresasexportadoras de uva, 2012 (Tesis de licenciatura). Arequipa, Perú: Universidad Católica de Santa María. Recuperado de: http://tesis.ucsm.edu.pe/ repositorio/handle/UCSM/4140.

Casolani, N. y Del Signore, A. (2016). Managers' opinions of factors influencing HACCP applications in Italian hotel/restaurant/café (HoReCa) sector. British Food Journal, 118(5), 1195-1207. doi: 10.1108/BFJ-09-2015-0312.

Castañeda, R., Fuentes, C. y Peñarrieta, M. (2016). Assessment of pre-requirements of HACCP and analysis of critical control points for safety during production of artisanal and industrial bread. Revista Boliviana de Química, 33(5), 196-208. Recuperado de: http://www.scielo. org.bo/pdf/rbq/v33n5/v33n5_a07.pdf.

Dal Prà, F. (2013). Studio sulla contaminazione microbiologica di carni macinate fresche. (Tesis de licenciatura). Padova, Italia: Università degli Studi di Padova. Recuperado de: http://tesi. cab.unipd.it/44748/.

El Comercio. (11 de Diciembre de 2015). Municipio de la Victoria clausuró locales de Plaza Vea y Metro. El Comercio, pág. 20.

Filippi, S. (2014). Elaborazione di un sistema informatizzato per la gestione dei punti critici di controllo in un'azienda alimentare(Tesis de licenciatura). Pisa, Italia: Università degli Studi di Pisa. Recuperado de: https://etd.adm.unipi. it/t/etd-02122015-105931/.

García, Y., Quiñones, L. y Parra, C. (2014). Análisis de las competencias laborales necesarias para la implementación del sistema HACCP en un restaurante. Retos Turísticos, 13(3), 113125. Recuperado de la base de datos Fuente Académica Premier de EBSCO.

Griffith, C., Jackson, L. y Lues, R. (2017). The food safety culture in a large South African food service complex: Perspectives on a case study. British Food Journal, 119(4), 729-743. doi: 10.1108/BFJ-11-2016-0533.

Gutiérrez, N., Pastrana, E. y Castro, J. (2011). Evaluación de prerrequisitos en el sistema HACCPen empresas delsectoragroalimentario.
Escuela de Ingeniería de Antioquia(15), 33-43. Recuperado de: http://www.scielo.org.co/pdf/ eia/n15/n15a04.pdf.

Hernández, R., Fernández, C. y Baptista, M. (2014). Metodología de la Investigación (Sexta ed.). México D.F., México: McGraw-Hill.

Lavilla, J. y Arizábal, R. (2011). Sistema HACCP y gestión de calidad en los restaurantes turísticos del distrito de Urubamba-Cusco. Caso: Tunupa Valley (Tesis de licenciatura). Cusco, Perú: Universidad Nacional de San Antonio Abad del Cusco. Recuperado de: http://repositorio. unsaac.edu.pe/handle/UNSAAC/915.

Ministerio de Salud (2002). Aprueban Reglamento Sanitario de Funcionamiento de Autoservicios de Alimentos y Bebidas. Lima, Perú: Ministerio de Salud. Recuperado de: https://www. saludarequipa.gob.pe/desa/archivos/Normas_ Legales/alimentos/RM_1653_2002.pdf.

Ministerio de Salud (26 de Mayo de 2016). Ministerio del Ambiente. Recuperado de: http://sinia. minam.gob.pe/documentos/politica-nacionalsalud-ambiental-2011

Mortimore, S. y Wallace, C. (2001). HACCP: Enfoque práctico (2 ed.). Zaragoza: Acribia S.A.

Muñoz, V. (2003). Programa educativo para la promoción y prevención de las enteroparasitosis, dirigido a las manipuladoras de alimentos de la ciudad de La Paz, gestión 2004(Tesis de maestría). La Paz, Bolivia: Universidad Mayor de San Andrés. Recuperado de: http://repositorio.umsa.bo/ handle/123456789/1188.

Organización de las Naciones Unidas para la Agricultura y la Alimentación. (2008). Manual de inspección de los alimentos basada en el riesgo. Roma, Italia: Servicio de Calidad de los Alimentos y Normas Alimentarias. Recuperado de: http://www.fao.org/3/a-i0096s.pdf.

Panisello, P. y Quantick, P. (2001). Technical barriers to Hazard Analysis Critical Control Point (HACCP). Food Control, 12(3), 165-173. doi: 10.1016/S0956-7135(00)00035-9.

Rodríguez, J., Pierdant, A. y Rodríguez, C. (2014). Estadística para administración [versión Adobe Digital Editions]. Recuperado de http:// ebookcentral.proquest.com.

Ruiz, C. (2003). Preparación higiénica de los alimentos (1 ed.). México: Trillas. 
Souza, D., Novo, I., Fernandes, E. y Kawamoto, L. (2016). Quality HACCP applied to flight catering. Independent Journal of Management \& Production, 7(5), 729-745. doi: 10.14807/ ijmp.v7i5.460.

Trafiałek, J. (2017). Implementation and functioning of HACCP principles in certified and noncertified food businesses: A preliminary study. British Food Journal, 119(4), 710-728. doi: 10.1108/BFJ-07-2016-0313.
Zapata, A. (2011). Condiciones de los servicios de alimentación y nutrición de los principales hospitales en atención infantil de la ciudad de Cochabamba en elaño 2011 (Tesis de maestría). Cochabamba, Bolivia: Universidad Mayor de San Simón. Recuperado de: http://atlas.umss. edu.bo:8080/jspui/handle/123456789/124. 
